identical. Arterial arrival time following treatment decreased by a mean of 0.49 seconds in the treatment group and increased by a mean of 0.12 seconds in the control group $(\mathrm{p}=0.0081)$.

Conclusion Preliminary results indicate that Sanguinate administered in the early acute phase of ischemic stroke improves $\mathrm{CBF}$ to the core infarct zone in experimental MCAO immediately following its administration.

Disclosures G. Christoforidis: 1; C; NIH grant. N. Saadat: None. M. Niekrasz: None. S. Roth: 1; C; NIH grant. T. Carroll: 1 ; C; NIH grant.

\section{E-022 IN VIVO ENDOTHELIALIZATION OF VEGF-COATED STENTS IN A RABBIT MODEL}

${ }^{1} \mathrm{~K}$ Panchendrabose*, ${ }^{2} \mathrm{~B}$ Har, ${ }^{3} \mathrm{~A}$ Mitha. ${ }^{1}$ Biomedical Engineering, University of Calgary, Calgary, $A B$, Canada; 'Libin Cardiovascular Institute, University of Calgary, Calgary, $A B$ Canada; ${ }^{3}$ Clinical Neurosciences, University of Calgary, Calgary, AB, Canada

\subsection{6/neurintsurg-2020-SNIS.58}

Introduction Implantation of cerebral stents are used in several disease conditions. Although stenting procedures offer many benefits, metal stents can elicit acute/subacute thrombosis and increase the chance of stroke. The risk of in-stent thrombus formation significantly decreases after formation of an endothelial cell layer over the stent, which typically takes several weeks to occur. One potential way to reduce the formation of thrombus is through biologic modifications that hasten endothelialization. In past studies, vascular endothelial growth factor (VEGF) has been shown to facilitate the recruitment and proliferation of cells that leads to endothelialization.

Methods In this study, VEGF was coated onto Solitaire stents using polylactic-co-glycolic acid (PLGA). Coated stents were deployed into the infrarenal abdominal aorta of New Zealand White rabbits for 72 hours $(n=5)$ (72h VEGF group) and compared to uncoated stents at 72 hours $(n=3)(72 \mathrm{~h}$ Control group) and seven days $(\mathrm{n}=3)$ ( $7 \mathrm{~d}$ Control group). Optical coherence tomography (OCT) was performed through the stented portion of the vessel, and representative images taken every five millimetres were used for analysis. Images were analyzed for neointimal area (stent area - lumen area), neointimal ratio ([stent area - lumen area]/stent area), minimal and maximal neointima thickness, stent-strut neointima coverage ratio (number of struts covered by a neointimal layer over the total number of struts), as well as thrombus area formation.
The analysis was performed by a cardiologist with clinical expertise in OCT imaging, who was also blinded to group allocation.

Results Post-deployment digital subtraction angiography demonstrated that VEGF-coated stents were deployed similar to uncoated stents, and without any obvious acute thrombus formation. Using two-tailed unpaired $t$-tests, the $72 \mathrm{~h}$ VEGF group showed significantly higher $(p<0.01)$ neointimal area and neointimal ratio compared to $72 \mathrm{~h}$ Control group. The minimal neointimal thickness of $72 \mathrm{~h}$ VEGF group was significantly higher $(p<0.05)$ than the $72 \mathrm{~h}$ Control group, but not statistically different from the $7 \mathrm{~d}$ Control group. There was a trend toward decreased thrombus formation in $72 \mathrm{~h}$ VEGF group versus the $72 \mathrm{~h}$ Controls $(p=0.07)$. The $7 \mathrm{~d}$ Controls had significantly higher stent-strut neointimal coverage ratio, neointimal area, neointimal ratio and maximum neointimal thickness compared to the $72 \mathrm{~h}$ VEGF group $(p<0.05)$ and $72 \mathrm{~h}$ Control group $(p<0.05)$.

Conclusion VEGF-coated stents are a promising biomodification to reduce secondary complications that may occur in the initial days following the procedure.

Disclosures K. Panchendrabose: None. B. Har: 3; C; Abbot. A. Mitha: 1; C; Stryker Neurovascular. 2; C; Microvention Inc., Cerus Endovascular, Stryker Corporation. 4; C; Fluid Biotech Inc.

\section{E-023 INTRAVENOUS OXYGEN CARRIER THERAPY DELAYS INFARCT SIZE PROGRESSION IN A CANINE LARGE VESSEL OCCLUSION MODEL}

${ }^{1,2}{ }^{2}$ King*, ${ }^{1} M$ Shazeeb, ${ }^{1} \mathrm{~J}$ Kolstad, ${ }^{1} \mathrm{C}$ Raskett, ${ }^{3} \mathrm{~J}$ Winger, ${ }^{3} \mathrm{~L}$ Kelly, ${ }^{1} \mathrm{Z}$ Vardar, ${ }^{1} \mathrm{~A}$ Kraitem, ${ }^{1} \mathrm{~V}$ Anagnostakou, ${ }^{3} \mathrm{~A}$ Krtolica, ${ }^{1} \mathrm{~N}$ Henninger, ${ }^{1} \mathrm{M}$ Gounis. ${ }^{1}$ Radiology, University of Massachusetts Medical School, Worcester, MA; ${ }^{2}$ Biomedical Engineering, Worcester Polytechnic Institute, Worcester, MA; ${ }^{3}$ Omniox Inx, San Carlos, CA

\subsection{6/neurintsurg-2020-SNIS.59}

Introduction Neuroprotective strategies represent a significant opportunity to delay infarct progression in patients suffering a Large Vessel Occlusion (LVO), allowing more time for treatment by mechanical thrombectomy. Oxygen carrier therapy has the potential to deliver oxygen to hypoxic tissues, allowing for a longer window of treatment before cell death.

Methods Twenty-three dogs were used in this study, with an additional 14 historical controls. Autologous clot was injected into the left MCA, confirmed on DSA, and the dog was transferred to a 3T MRI. In order to determine infarct evolution rate, Perfusion Weighted Imaging was performed and relative Time to Peak (rTTP) maps were generated. ${ }^{1}$ Based on the initial infarct evolution pathway (slow or rapid progressors), animals were randomized to either receive oxygen carrier or vehicle control. Regardless of evolution pathway, or treatment group, IV bolus was given 45 minutes after clot placement. Following bolus, diffusion weighted imaging (DWI) was performed every 30 minutes to assess infarct evolution. After 5 hours, dogs were removed from the MR, euthanized and the brain was explanted for histological processing. To calculate the true infarct volume, apparent diffusion coefficient (ADC) was calculated from the DWI images, and a threshold value of $0.533 \times 10^{-3} \mathrm{~mm}^{2} / \mathrm{s}$ was used to differentiate infarct. The predicted final infarct size was taken from rTTP maps as the area with greater than $4.5 \mathrm{~s}$ delay and used to normalize infarct volume. 


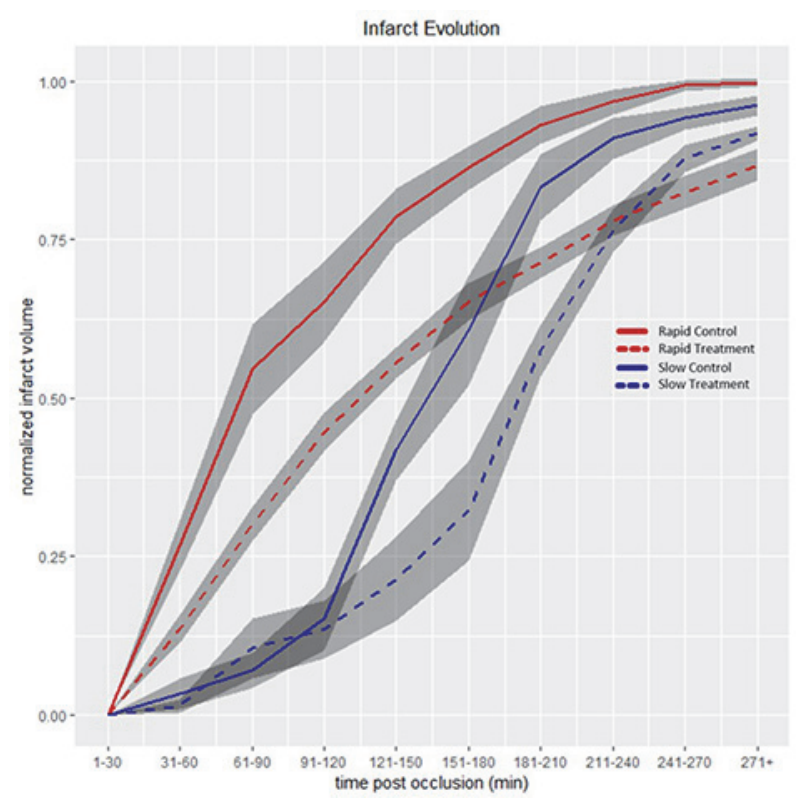

Abstract E-023 Figure 1 Infarct evolution curves based on DWI lesion size for the four groups all data has been normalized to the rTTP total infarct size. Gray area represents the standards error of the mean

Results Of the 23 dogs used in the active group, 5 were excluded due to spontaneous recanalization or lack of sufficient infarct on DWI. From the included 18 dogs, 11 were identified as rapid progressors, and 7 as slow. The figure shows the average infarct growth rate for the four groups, showing the oxygen carrier increasing the time needed for the infarct to reach $50 \%$ of its expected size by approximately 45 minutes in the rapid group, and 30 minutes in the slow. Overall the final infarct size was reduced in both the rapid and slow groups, 0.99 vs 0.87 control versus treatment, and 0.97 vs 0.92 control versus treatment, respectively $(\mathrm{p}<0.001$ and 0.022 ).

Conclusions Oxygen carrier therapy shows promise to slow down the infarct growth after an LVO, allowing for more time to perform mechanical thrombectomy. Not only was the time to $50 \%$ infarct increased, but after 5 hours, the infarct in the oxygen carrier groups still showed an area of penumbra.

\section{REFERENCE}

1. Trans/ Stroke Res. 2019 Sep 3.

Disclosures R. King: None. M. Shazeeb: None. J. Kolstad: None. C. Raskett: None. J. Winger: 5; C; Omniox Inc. L. Kelly: 5; C; Omniox Inc. Z. Vardar: None. A. Kraitem: None. V. Anagnostakou: None. A. Krtolica: 5; C; Omniox Inc. N. Henninger: 1; C; K08NS091499. M. Gounis: None.

\section{E-024 SPONTANEOUS VERTEBRAL ARTERIOVENOUS FISTULA MIMICKING BRACHIAL RADICULO-PLEXOPATHY: A CASE REPORT}

${ }^{1} \mathrm{~A}$ Larson*, ${ }^{1} \mathrm{~L}$ Rinaldo, ${ }^{2} \mathrm{C}$ Arnold Fiebelkorn, ${ }^{2} \mathrm{~N}$ Young, ${ }^{1} \mathrm{G}$ Lanzino. ${ }^{1}$ Neurosurgery, Mayo Clinic, Rochester, MN; ${ }^{2}$ Neurology, Mayo Clinic, Rochester, MN

10.1136/neurintsurg-2020-SNIS.60
Vertebral arteriovenous fistulas (VAVF) are uncommon, highflow communications between a vertebral artery and surrounding venous plexus that occur spontaneously or secondary to trauma. A 57-year-old female presented with a multi-day history of rapidly progressive numbness and weakness in the left C5-C6 dermomyotomes. Her physical exam findings and subsequent electrophysiological testing were suggestive of a brachial radiculo-plexopathy. Noninvasive imaging demonstrated venous congestion with multilevel compromise of the left-sided cervical foramina, and subsequent vertebral angiography confirmed a VAVF, which was treated with trapping of the involved VA segment. Her numbness and weakness progressively improved with concurrent involution of the dilated veins. An exceptional case of VAVF manifesting as a brachial radiculo-plexopathy is presented. VAVF are rare, though they may be considered as a potential underlying cause in patients with comparable symptoms. Endovascular embolization has been demonstrated as a safe and efficacious method in treating VAVFs, though multiple patient-specific factors must be contemplated.

Disclosures A. Larson: None. L. Rinaldo: None. C. Arnold Fiebelkorn: None. N. Young: None. G. Lanzino: None.

\section{E-025 TRANSORBITAL ENDOVASCULAR EMBOLIZATION OF CAROTID-CAVERNOUS FISTULAS: A CASE SERIES}

B King*, J Steinberg, A Wali, R Rennert, D Santiago-Dieppa, J Pannell, A Khalessi, S Olson. University of California San Diego, La Jolla, CA

\subsection{6/neurintsurg-2020-SNIS.61}

Introduction/Purpose Carotid-cavernous fistulas (CCFs) are typically managed by embolization, with varied approaches described. Direct transorbital venous access may be utilized if anatomic constraints limit fistulous access via standard venous or arterial access. We present eight cases of successful CCF obliteration through direct transorbital puncture of the cavernous sinus or through indirect cannulation via the superior or inferior ophthalmic veins.

Materials and Methods Patient data was gathered through retrospective chart review from August 2017 to December 2019. Demographics, fistula type, treatment method, obliteration status, and complications were recorded.

Results Eight patients (M:4, F:4, age $44 \pm 15$ years) were identified who underwent a transorbital approach for treatment of CCF. Six CCFs were spontaneous, and two were deemed to be traumatic in nature following motor vehicle accidents. One patient had a direct fistula (Barrow type A), while the remainder had indirect fistulas (Barrow types B, C, D). Three patients underwent a direct transorbital embolization; one underwent transarterial embolization followed by transorbital embolization; one underwent transarterial embolization, attempted transvenous embolization, followed by a direct transorbital embolization; one underwent attempted transvenous embolization followed by direct transorbital embolization; and two underwent transarterial embolization, followed by transvenous embolization, followed by direct transorbital embolization.

Fistulous occlusion was achieved in all patients following transorbital embolization. Seven patients demonstrated complete resolution of ophthalmic symptoms with normalization of intraocular pressures. One of these patients required an immediate post-operative lateral canthotomy due to transient 